\title{
Analytic solutions of the PRISM equations for Gaussian polymers
}

\author{
Matthias Fuchs* \\ Department of Materials Science and Engineering, University of Illinois, 1304 West Green Street, Urbana, IL 61801, USA
}

Received: 1 January 1997

\begin{abstract}
The polymer reference interaction site model (PRISM) equations for the collective structure of macromolecular liquids are simplified using the Wiener-Hopf factorization technique. This approach is expected to be useful in systems exhibiting strongly different length scales. As an application, melts and solutions of Gaussian flexible polymers are considered. It is shown that PRISM theory in agreement with experiments predicts liquid like packing for high polymer concentrations and mean-field like scaling laws for the dilute to semidilute crossover at the $\Theta$-temperature. In a systematic expansion the range of validity and the leading corrections to the low density scaling law description are calculated.
\end{abstract}

PACS: $21.25 . \mathrm{hq}$

\section{Introduction}

Liquids consisting of macromolecules possess intra- and intermolecular correlations which are characterized by length scales of greatly varying magnitude. Significant progress in the theoretical understanding of, especially, the long-wavelength phenomena has been made by the use of scaling, Renormalization Group and self-consistent-field techniques [1-4]. Lattice models also have been used to address dense polymeric systems, which are dominated by short ranged intermolecular interactions [5, 6]. Although of interest, it has as yet been difficult to find an unified framework encompassing the various concentration ranges of polymeric systems, dilute, semidilute, and concentrated solutions and melts, which also provides reasonable results on microscopic and macroscopic length scales.

Schweizer and Curro have introduced a liquid state theory approach to the structure of polymeric fluids [7]. This integral equation theory for macromolecules (PRISM) is an extension to flexible polymers of the reference interaction

Dedicated to Wolfgang Götze on the occasion of his 60th birthday

* Present address: Physik Department, Technische Universität München, James-Franck-Strasse, D-85747 Garching, Germany site model (RISM) of Chandler and Andersen [8] for rigid small molecules. Its applicability to dense, semiflexible polymer melts has been verified e.g. by comparison with computer simulations [9], and its value for the description of chain polymer melts, melts of star polymers, blends, block copolymer systems, nonuniform polymer liquids, and polymer crystallization is reviewed in $[10,11]$.

In this paper, we show by an exact solution of the PRISM integral equations for a simplified intramolecular structure that PRISM qualitatively correctly describes the collective structure at low and high densities at the $\Theta$-temperature. On the one hand, the intermolecular excluded volume interactions screen long ranged intramolecular correlations in melts and dense solutions, and small compressibilities result $[10,11]$. On the other hand, for lower densities the steric interactions and macromolecular connectivity compete and lead to enhanced collective density fluctuations correlated over macroscopic length scales. PRISM correctly predicts universal scaling laws for the dilute to semidilute crossover close to the (tricritical) $\Theta$-point $[12,13]$. In this calculation, we use for $\Theta$-solutions, as holds for concentrated solutions and melts, that the intramolecular structure on long length scales is appropriately described by a Gaussian approximation $[1,5]$. The present rigorous systematic expansion of the PRISM integral equations extends the knowledge of the related "thread" and "string" PRISM approximations [14, 15] as it allows to identify the range of validity of the scaling law description and to calculate corrections.

Results from non-linear integral equations can be difficult to obtain, even numerically, if greatly varying length scales are encountered; note that in the cases considered a solution in the limit of divergent correlation lengths is aspired, which also rigorously enforces the excluded volume constraint microscopically. Thereto, in Sect. 3 the RISM or PRISM integral equations with the atomic mean spherical approximation (MSA), listed in Sect. 2, are reformulated using the Wiener-Hopf factorization introduced by Baxter [16]. This section is kept general as the reformulation is expected to be useful in further applications of PRISM theory to systems which exhibit large separations of the relevant length scales. It includes as special limits the hard sphere mixtures studied by Baxter [16], the atomic systems 
of particles interacting with Coulombic and Yukawa potentials treated by Høye et al. [17, 18], and the RISM treatment of diatomic molecules of Morris and coworkers [19]. The critical point properties of such integral equations have for example been discussed for atomic one $[20,21]$ and two $[22,23]$ component liquids, where divergent compressibilities mathematically result from non-linearities introduced by interatomic pair potential tails. Intramolecular correlations, as present in RISM and PRISM equations, lead to similar mathematical non-linearities as noted in [19]; there approximations prevented a closer study. In Sect. 4 of this contribution and in the appendix, a detailed analytical study of the resulting macroscopic correlations for flexible Gaussian homopolymer systems is presented, and inter alia mean-field like scaling laws are derived. The discussion in Sect. 5 summarizes our findings.

\section{The RISM integral equations}

The reference interaction site model (RISM) equations of Chandler and Andersen [8] are generalizations of the Ornstein-Zernicke equations of monoatomic [24] to molecular liquids. Starting from the intramolecular structure and the intermolecular potentials, $V^{i j}(r)$, the collective liquid structure is calculated. The intramolecular structure functions shall be denoted by $\hat{\omega}^{i j}(k)$, where the indices $i, j$ may either label different species of molecules or sites on a single macromolecule. Their diagonal elements approach the self-scattering value for large wave vectors, $\hat{\omega}^{i j}(k) \rightarrow \delta^{i, j}$ for $k \rightarrow \infty$. For monoatomic particles, this results holds for all $k$.

In order to extend the small rigid-molecule RISM equations to flexible macromolecules, Schweizer and Curro [7, $10,11]$ have introduced the polymer RISM (PRISM) equations, where, in the most simple case, the sites are equivalent and a macromolecule of $N$ segments can be characterized by its (independently measurable) collective intramolecular structure factor, $\hat{\omega}(k)={ }_{N}^{1} \sum_{\alpha, \beta=1}^{N} \hat{\omega}^{\alpha \beta}(k)$.

The partial structure factors of the macromolecular liquid, which in principle are measurable by scattering experiments, are determined by the intramolecular structure and the site-site total intermolecular correlation function, $\hat{h}^{i, j}(k)$ :

$\hat{S}(k)=\varrho \hat{\omega}(k)+\varrho \hat{h}(k) \varrho$,

where matrix notation is used and carets denote 3-dimensional Fourier transforms. The total correlation function, $h^{i j}(r)$, is connected to the intermolecular site-site radial distribution or pair correlation function, $h^{i j}(r)=g^{i j}-1$. The diagonal matrix, $\varrho^{i j}=\delta^{i j} \varrho^{i}$, consists of the segmental densities, $\varrho^{i}$. The Ornstein-Zernicke like (P)-RISM equation connects $\hat{S}(k)$ to the direct correlation function, $c^{i j}(r)$ :

$\hat{S}^{-1}(k)=\hat{\omega}^{-1}(k) \varrho^{-1}-\hat{c}(k)$.

Intermolecular excluded volume repulsion is taken into account by requiring that the probability of segments on different molecules to overlap vanishes:

$g^{i j}(r)=0 \quad$ for $r<R^{i j}=\frac{1}{2}\left(R^{i}+R^{j}\right)$, where the $R^{i}$ are (effective) hard core diameters of the monomers.

Extending work on atomic liquids, closure relations are formulated for the direct correlation function, $c^{i j}(r)$, which generally express the expectation that the spatial range of $c^{i j}(r)$ is small and close to the one of the actual monomeric pair potential, $V^{i j}(r)[8,10,11,24]$. For the repulsive steric interactions the Percus-Yevick (PY) closure is rather accurate, and the mean spherical approximation (MSA) is a useful extension to handle tail potentials [8, 24]. The MSA closure on the monomeric level is:

$c^{i j}(r)=-\beta V^{i j}(r) \quad$ for $r>R^{i j}$,

where $\beta$ is the inverse temperature. The results for the coherent or intermolecular liquid structure are now determined if the pair potentials, $V^{i j}(r)$, and the intramolecular structures, $\hat{\omega}^{i j}(k)$, are specified. We will consider situations where the assumption of a given intramolecular structure is justified and chose simple expressions for the $\hat{\omega}^{i j}(k)$, which allow analytical solutions of Eqs. (1) to (4). Section 4 presents such a calculation where the general frame work of Sect. 3 is put to use.

\section{Method of solution}

The Wiener-Hopf factorization as pioneered by Baxter allows to transform the integral equations (1) to (4) into possibly simpler equations for a factor function, $Q^{i j}(r)$. To this end it is convenient to define a short ranged direct correlation function, $c_{0}^{i j}(r)$, by:

$c_{0}^{i j}(r)=c^{i j}(r)+\beta V^{i j}(r)$,

which satisfies:

$c_{0}^{i j}(r)=0 \quad$ for $r>R^{i j}$.

Also the non-trivial part of the intramolecular correlations shall be split off by:

$\hat{w}^{i j}(k)=\hat{\omega}^{i j}(k)-\delta^{i j}$.

Its Fourier backtransform exits because of $\hat{w} \rightarrow 0$ for $k \rightarrow$ $\infty$.

$w^{i j}(r)=\int \frac{d k}{2 \pi} e^{-i k r} \hat{w}^{i j}(k) \quad$ for $r>0$,

where it is convenient to define $w^{i j}(-r)$ by analytic continuation.

Following Baxter [16] the Wiener-Hopf factorization is written as

$\hat{S}^{-1}(k)=\hat{Q}^{T}(-k) \hat{Q}(k) \varrho^{-1}$.

$\hat{Q}^{i j}(k)$ is given by the Fourier transform of the factor function $Q^{i j}$ :

$\hat{Q}^{i j}(k)=\delta^{i j}-\int d r e^{i k r} Q^{i j}(r)$.

Important properties of $Q^{i j}(r)$ can be deduced from (2) and (7), and the known properties of $\hat{\omega}(k), \hat{V}(k)$ and the short range behavior of $c_{0}(r)$ in (5) $[16,17,18] . Q^{i j}(r)$ vanishes for $r$ smaller than $\lambda^{i j}$, where 
$\lambda^{i j}=\frac{1}{2}\left(R^{i}-R^{j}\right)$,

and can be split into an internal $\left(r<R^{i j}\right)$ and an external part (using the Heaviside step function $\theta(r)$ ):

$Q^{i j}(r)=\theta\left(r-\lambda^{i j}\right)\left[\theta\left(R^{i j}-r\right) q^{i j}(r)+\bar{Q}^{i j}(r)\right]$.

From the continuity of $Q^{i j}(r)$ at $R^{i j}$ follows [16]:

$q^{i j}\left(R^{i j}\right)=0$.

As has been shown in $[17,18]$, the external part, $\bar{Q}^{i j}(r)$, can be obtained from the known non-analyticities of $\hat{\omega}(k)$ and $\hat{V}(k)$ in the lower half plane, $\Im k<0$.

$$
\begin{gathered}
\bar{Q}(r)=-\int \frac{d k}{2 \pi} e^{-i k r}\left(\hat{Q}^{T}(-k)\right)^{-1}\left[\hat{\omega}^{-1}(k)+\beta \hat{V}(k) \varrho\right] \\
\text { for } r>\lambda^{i j},
\end{gathered}
$$

where $Q^{-1}(-k)$ is regular for $\Im k<0$. The consequences of the external part, $\bar{Q}(r)$, of the factor function have been discussed for cases where it results from the poles of $\hat{V}(k)$ in the lower half plane $[17,18,21]$. In RISM calculations for diatomic molecules it was recognized that the intramolecular structure, i.e. $\hat{\omega}^{-1}(k)$ in (12), may also cause $\bar{Q}^{i j}(r) \neq 0$ [19]. There, however, a zero or few pole approximation had to be performed which prevented its study and even lead to spurious divergences for low densities. In this work, we will focus on physical effects resulting from the intramolecular correlations which requires an exact treatment of (12). Thereto, we use simplified models for the intramolecular structure, $\hat{\omega}(k)$, from the outset, which, on the one hand, are qualitatively adequate, and, on the other hand, allow to evaluate (12).

If the external part of the factor function vanishes, the Wiener-Hopf factorization leads to linear algebraic equations for the coefficients of the internal part [16]. In the general case, either if external potential tails or if intramolecular correlations lead to finite $\bar{Q}$, non-linear algebraic solvability conditions result from (12) [18]. Assuming a discrete set of poles of $\left[\hat{\omega}^{-1}(k)+\beta \hat{V}(k) \varrho\right]$ at the positions $k=-i \kappa_{n}$, where $\kappa_{n}>0$, the functional form of $\bar{Q}(r)$ is known to be a sum of exponentials [18].

$\bar{Q}^{i j}(r)=\sum_{n} \bar{Q}_{n}^{i j} e^{-\kappa_{n} r}$.

Via (12), the coefficients of these exponentials in the external part of the factor function are connected to $\hat{Q}(-k)$ at the position of the poles, $k=-i \kappa_{n}$, i.e. the Laplace transforms of the factor function itself:

$\tilde{Q}^{i j}\left(\kappa_{n}\right)=\int_{\lambda^{i j}}^{\infty} d r e^{-\kappa_{n} r} Q^{i j}(r)$,

where the known residues of the intramolecular and potential functions enter.

$\left[1-\tilde{Q}^{T}\left(\kappa_{n}\right)\right] \bar{Q}_{n}=i \operatorname{Res}\left[\hat{\omega}^{-1}(k)+\beta \hat{V}(k) \varrho,-i \kappa_{n}\right]$

In the case of attractive Yukawa tail potentials and the MSA closure it is (15) which leads to a quartic equation allowing for special solutions like a critical point [21].

The further solution for $Q(r)$ progresses from (1) and (7) via a Fourier backtransformation and the observation that $\left(Q^{T}(-k)\right)^{-1}$ is non-singular in the lower half plane, $\Im k<0$
$[16,18]$. The form of the factor function, Eqs. (10) and (13), can be used and leads to the appearance of the parameters $\tilde{g}$, which are the Laplace transforms of $g(r)$ at the poles in (12), $\kappa=\kappa_{n}>0$ :

$\tilde{g}^{i j}(\kappa)=\int_{0}^{\infty} d r e^{-\kappa r} r g^{i j}(r)$.

Obviously, for each pole in (12) one is left with two (sets) of unknowns, $\bar{Q}_{n}$ and $\tilde{g}\left(\kappa_{n}\right)$, and one (set) of equations, i.e. Eq. (15).

Inside the excluded radial range, one obtains an integrodifferential equation for $Q(r)$ which allows to determine the functional form of the internal part $q(r)[16,18]$ :

$$
\begin{aligned}
& \frac{d}{d r} Q^{i j}(r)+\sum_{l} \int_{\lambda^{i l}}^{\infty} d s Q^{i l}(s) \\
& \quad \times\left[(r-s) 2 \pi \varrho^{j}+\frac{d}{d r} W^{l j}(|r-s|)\right] \\
& =2 \pi \varrho^{j} r-2 \pi \varrho^{j} \sum_{l} \sum_{n} \bar{Q}_{n}^{i l} e^{-\kappa_{n} r} \tilde{g}^{l j}\left(\kappa_{n}\right)+\frac{d}{d r} W^{i j}(r) \\
& \quad \text { for } \lambda^{i j}<r<R^{i j} .
\end{aligned}
$$

For hard sphere mixtures, Eq. (17) alone determines $q(r)$ as both $\bar{Q}(r)$ and $W(r)$ vanish [16]. If tail potentials are present in atomic liquids, Eq. (17) still simplifies as $W(r)=0$ $[17,18]$. Yet, Eqs. (15) and (17) then do not suffice to determine all coefficients entering in the factor function $Q(r)$. Another set of equations for the Laplace transforms of the pair correlation functions is required. It can be found from analytically extending the solution $q(r)$ of (17) to $r>R^{i j}$ and subtracting it from the full equation for $r>R^{i j}$ [18]. As a first result, the contact value of the site-site intermolecular pair correlation function follows [18]:

$g^{i j}\left(R^{i j}\right)=\left.\frac{1}{2 \pi \varrho^{j} R^{i j}} \frac{d}{d r} q^{i j}(r)\right|_{r=R^{i j}}$.

Moreover, another equation for the Laplace transforms, $\tilde{g}$ Eq. (16), is found [18]:

$\sum_{l}\left[\delta^{i l}-\tilde{Q}^{i l}(\kappa)\right] 2 \pi \varrho^{j} \tilde{g}^{l j}(\kappa)=\kappa \sum_{l} \check{q}^{i l}(\kappa) \breve{w}^{l j}(\kappa)$,

where

$\breve{q}^{i j}(\kappa)=\int_{R^{i j}}^{\infty} d r e^{-\kappa r} q^{i j}(r)$,

and

$\check{w}^{i j}(\kappa)=\delta^{i j}+\int_{0}^{\infty} d r e^{-\kappa r}\left(w^{i j}(r)-w^{i j}(-r)\right)$.

Equation (19) provides the last set of linear equations to determine the coefficients of the factor function, $Q(r)$.

Summarizing, the method of solution proceeds via, first, determining the functional form of $\bar{Q}(r)$ from (12) and of $q(r)$ from (17). Sets of linear equations for the coefficients in $Q(r)$ result from (17) and (19), while the $\tilde{g}\left(\kappa_{n}\right)$ are treated as parameters. Non-linear equations for them finally follow from the solvability condition (15).

Besides the contact value, see (18), the compressibility, $\chi_{T}$, where $\beta / \chi_{T}=\sum_{i j} \varrho^{i}\left(\hat{S}^{-1}(0)\right)^{i j} \varrho^{j}$ [24], can easily be obtained from $Q(r)$. From (7): 


$$
\frac{\beta}{\chi_{T}}=\sum_{i l j} \varrho^{i} \hat{Q}^{l i}(0) \hat{Q}^{l j}(0) .
$$

Also, the 3-dimensional Fourier transform of the total correlation function, $\hat{h}^{i j}(k)$ in (1), and thereby the structure factors can be calculated from the Laplace transform of the pair correlation function, $\tilde{g}(\kappa)$ Eq. (16), evaluated at the Laplace variable $\kappa=-i k[18]$ :

$$
\hat{S}^{i j}(k)=\varrho^{i} \hat{\omega}^{i j}(k)+4 \pi \varrho^{i} \varrho^{j} \Re\left\{\frac{1}{\kappa} \tilde{g}^{i j}(\kappa)-\left.\frac{1}{\kappa^{3}}\right|_{\kappa=-i k}\right\} .
$$

In general, the pair correlation function $g(r)$ itself can be obtained by numerical means only.

An analytical solution of the PRISM equations in most cases cannot compete with numerical techniques if accurate modeling of the intra- and intermolecular interactions is required. However, if rigorous results for the long wavelength properties of the PRISM equations are aspired, simplified models for $\hat{\omega}(k)$ can capture the essential aspects of the intramolecular structure and may allow to study in detail the consequences of macromolecular connectivity, self similar internal structures and of steric interactions; see the next section where this is done for one-component Gaussian polymer systems.

\section{Gaussian polymer systems with intermolecular excluded volume interactions}

It is well established that the mesoscopic aspects of the single polymer conformations in a homopolymer melt or in concentrated solutions can be described by Gaussian statistics $[1,5]$. It is possible to use this observation ("Flory's ideality hypothesis") in the RISM integral equations, and to argue further that chain end effects are negligible for large degrees of polymerization, $N$ [7]. The resulting most simple version of the PRISM equations then reduces to scalar equations which are uniquely determined if the single polymer structure factor, $\hat{\omega}(k)$, and the intermolecular monomeric pair potential, $V(r)$, are specified. The segmental density, $\varrho$, enters and is often reported in terms of a packing fraction, $\Phi=\pi \varrho \sigma^{3} / 6$, where the Gaussian segment size, $\sigma$, is used as length scale.

From the theories of simple liquids [24], it is well known that the structure of dense liquids is dominated by the intermolecular excluded volume or hard core impenetrability constraint. In order to study the interplay of intermolecular packing and intramolecular connectivity it therefore suffices to consider the steric interactions only and neglect the pair potential tails, i.e. $V(r)=0$ is set. Recently, it has been shown that in polymer solutions, where the polymer and solvent molecules interact with purely steric repulsion only, the different kinds of solvent qualities can result from different chemical potentials of the solvent molecules [25]. A $\Theta$-solvent, where the intramolecular structure of a polymer is Gaussian on long length scales, therefore can be achieved for monomers which have no potential interaction except for the excluded volume one. The simple version of the PRISM theory studied here, whose applicability to dense polymeric systems has been tested $[9,10,11]$, therefore can also be applied to polymer solutions at the $\Theta$-temperature. The question, whether PRISM achieves a correct description of the
$\Theta$-line for all densities, from the dilute solution to the melt, shall be addressed in this section.

For $V(r)=0$ in (4), PY and MSA closure agree, and $c(r)=0$ for $r>R$, where $R$ is the effective hard sphere diameter of the monomers, i.e. $g(r)=0$ for $r<R$. Note that the hard core problem considered here can be treated with such an atomic closure [10], whereas attractive potential tails require more complex molecular closures [26]

The collective structure and the intermolecular correlations of the simple homopolymer system are determined by the PRISM equations, Eqs. (1) to (4), and the intramolecular structure factor, $\hat{\omega}(k)$. The usual normalization, $\hat{S}(k)=$ $\hat{\omega}(k)+\varrho \hat{h}(k)$, shall be chosen in this section (instead of Eq. (1)). As a consequence of the connectedness of the macromolecule, $\hat{\omega}(k)$ counts the number of monomers for small wave vectors, $\hat{\omega}\left(k \ll R_{g}\right) \rightarrow N\left(1-{ }_{3}^{1} k^{2} R_{g}^{2}\right)$, where the radius of gyration, $R_{g}$, is a measure of the size of the molecule. In an intermediate range, flexible Gaussian polymers are self-similar, which leads to a power law behavior, $\hat{\omega}(k) \rightarrow \frac{12}{k^{2} \sigma^{2}}$ for $1 / R_{g} \ll k \ll 1 / \sigma$. For larger wave vectors, the self scattering contribution remains in $\hat{\omega}(k), \hat{\omega}(k)$ $\rightarrow 1$ for $k \rightarrow \infty$. The statistical segment size $\sigma$, can be defined from $\sigma^{2}=\lim _{N \rightarrow \infty} 6 R_{g}^{2} / N$ [1]. Its connection to microscopic lengths depends on chemical aspects of the polymer [5]. Our model thus contains one microscopic parameter, an effective aspect ratio, $\Gamma=\sigma / R$. Flexible polymers whose global macromolecular structure is Gaussian differ in the chemistry dependent value of the aspect ratio which, e.g., lies between $0.8<\Gamma<1.3$ for various polyolefins [27].

A handy interpolation form for $\hat{\omega}(k)$ which contains the mentioned limits and exhibits small quantitative errors in the intermediate ranges is given by [28]:

$\hat{\omega}(k)=\frac{N}{1+k^{2} \xi_{c}^{2}}+1, \quad$ where $\quad \xi_{c}^{2}=R_{g}^{2} / 2=\frac{N \sigma^{2}}{12}$.

Importantly, it allows to solve the PRISM equations without further approximations. From previous solutions of the RISM equations it is known, that spurious and unphysical divergences may result if approximations during the WienerHopf factorization are required [19].

The intramolecular correlations in (12) lead to a finite external factor function, $\bar{Q}(r)$, outside the hard sphere diameter, $R$, as $\hat{\omega}^{-1}(k)$ has a pole in the lower half plane at:

$k=-i z, \quad$ where $\quad z^{2}=\frac{12}{\sigma^{2}}\left(1+\frac{1}{N}\right)$.

Due to this pole, the functional form of $\bar{Q}(R)$ is:

$\bar{Q}(r)=d e^{-r z}$.

It can be inserted in (15) and with the known residue of $\hat{\omega}^{-1}$ at $k=-i z$ leads to the solvability condition of our model, Eq. (A.1), given in the appendix. The Laplace transforms, Eqs. (14) and (16), of the factor function and of the pair correlation function at the inverse length scale $z$, from (25), are required in the following and will be abbreviated by: $\tilde{Q}=\tilde{Q}(\kappa=z)$ and $\tilde{g}=\tilde{g}(\kappa=z)$.

The functional form of $q(r)$, which completes $Q(r)$ via (10), is found by applying $\left(\left(\frac{d}{d r}\right)^{2}-\left(1 / \xi_{c}\right)^{2}\right)$ to (17). Denoting derivatives by a prime, one finds: 


$$
\begin{array}{r}
q^{\prime \prime \prime}(r)-z^{2} q^{\prime}(r)=-\frac{2 \pi \varrho}{\xi_{c}^{2}}[ \\
\quad\left(z^{2} \xi_{c}^{2}-1\right) \tilde{g} d e^{-z r}+r \\
\left.-\int_{0}^{\infty} d s(r-s) Q(s)\right]
\end{array}
$$

Satisfying the condition, Eq. (11), the solution $q(r)$ of (27) is:

$$
\begin{aligned}
q(r)= & \frac{a}{2}\left(r^{2}-R^{2}\right)+b(r-R)+c\left(r e^{-z r}-R e^{-z R}\right) \\
& +e\left(e^{-z r}-e^{-z R}\right)+f\left(e^{z r}-e^{z R}\right)
\end{aligned}
$$

Comparing the form (28) to the known results for hard spheres, $Q^{\mathrm{hs}}=q^{\mathrm{hs}}=a^{\prime} / 2\left(r^{2}-R^{2}\right)+b^{\prime}(r-R)$ [16], and to the results including a Yukawa potential tail, $q^{\mathrm{Yu}}=$ $q^{\mathrm{hs}}+e^{\prime}\left(e^{-z^{\prime} r}-e^{-z^{\prime} R}\right)$ and $\bar{Q}^{\mathrm{Yu}}(r)=d^{\prime} e^{-r z^{\prime}}$ [18], one notices that the intramolecular correlations lead to a more complex structure of the factor function inside the hard core diameter. The considered problem goes back to the PY treatment of hard spheres in the limit of $N=0$ [16], and thus a generalization of the useful hard sphere relation for the compressibility holds, $\hat{S}(0)=\varrho \chi_{T} / \beta=(2 \pi \varrho / a(N+1))^{2}$.

The set of six linear equations for the coefficients $a, \ldots$, $f$ of the factor function is listed in the appendix, Eqs. (A.2) to (A.7). It can be solved using symbolic manipulation software packages but the solutions, depending on the still unknown parameter $\tilde{g}$, the packing fraction, $\Phi$, the aspect ratio, $\Gamma$, the Gaussian segment size, $\sigma$, and the degree of polymerization, $N$, are too long to report. When the solutions $a(\tilde{g}), \ldots, f(\tilde{g})$ are entered into the solvability condition, Eq. (A.1), a quartic equation for $\tilde{g}$ follows. Its solutions and the results for the collective liquid structure shall be discussed for three limits of special interest in the rest of this section; the algebra involved is sketched in the appendix.

\subsection{Noninteracting case}

The limit of vanishing hard sphere diameter of the monomers, i.e. $R=0$, is trivial as it turns off the only intermolecular interactions in the studied system, the excluded volume interaction. Not surprisingly therefore, ideal gas like results are found:

$\hat{S}(k)=\hat{\omega}(k), \quad g(r)=1 \quad$ for $R=0$.

For non-interacting macromolecules, the collective density fluctuations arise from an incoherent superposition of the single polymer contributions, and the intermolecular correlations are random. Trivially, the same wave vector dependent compressibility, $\hat{S}(k)=\hat{\omega}(k)$, is found in the limit of vanishing density, $\varrho=0$. Note, that in this limit the pair correlation function is different, $g(r) \neq 1$ for $\varrho=0$, as it describes two molecule interactions.

These (trivial) limits serve as convenient starting points to identify the physical solutions in the general case. Moreover, the limit of vanishing hard core diameter stresses that a finite excluded volume is required to build up interparticle correlations in the studied $(V(r)=0)$ system. This should be kept in mind for the case studied in Sect. 4.3 below.

\subsection{Infinite degree of polymerization}

The limit of infinite molecular weight, $1 / N=0$, is of interest as in this case the intramolecular correlations are selfsimilar on all length scales beyond microscopic distances, and thus the intramolecular structure factor exhibits a true small $k$ divergence, $\hat{\omega}(k) \sim 1 / k^{2}$ for $k \sigma \ll 1$. It has long been realized that intermolecular interactions screen this divergence and that the collective structure exhibits finite (in melts even small) density fluctuations $[1,3,5]$. PRISM theory correctly captures this correlation hole effect $[7,14,15]$, as has been demonstrated by numerical work and in certain approximations. Rigorously, it can be shown for the studied intramolecular structure, as is detailed in the appendix. Important in this context is (22) which allows to determine the compressibility directly and circumvents limiting procedures like $k \rightarrow 0$. Thus, except for in the single polymer limit, PRISM predicts finite collective density fluctuations of flexible $(\Gamma \approx 1)$ polymers with steric interactions in the accessible density range.

The equations for the Wiener-Hopf factor function, $Q(r)$, simplify somewhat in the case $1 / N=0$, and a typical solution for the collective structure factor in the melt case is reproduced in Fig. 1. The packing fraction is chosen rather high, $\Phi=1.0$, in order to observe at infinite $N$ the familiar dense packing and liquid like structure [24]. At high densities, $\hat{S}(k)$ is dominated by the excluded volume constraints even for smooth interaction potentials [24]. The infinite molecular weight result compares well to a solution for finite $N$, where $N=10^{3}$ corresponds to a molecular weight of the order $M \approx 10^{5}-10^{6}$, and $N \approx 10^{5}$ can be achieved in practice $[1,5]$. A small but finite compressibility at low wave vectors justifies the incompressibility approximation, $\hat{S}(k) \approx 0$ for $k \sigma<1$, which is used widely in polymer theories [1]. It should be noted, however, that the studied polymeric liquid exhibits larger compressibilities than a simple atomic liquid [24], where packing at this high packing fraction even is impossible for e.g. hard spheres. One also concludes that the packing fraction in our model has to be corrected for intramolecular overlap of the monomers in order to calculate the actual sterically excluded volume; see [14].

The inset in Fig. 1 compares the used $\hat{\omega}(k)$ with the discrete chain generalization of the Debye function, $\hat{\omega}^{G}$, which results for a polymer chain which is Gaussian on all length scales $[1,28]$ :

$$
\begin{aligned}
\hat{\omega}^{G}(k)= & \frac{1}{N} \sum_{\alpha \beta} e^{-\frac{k^{2} \sigma^{2}}{6}|\alpha-\beta|}=\frac{1-f^{2}-2 f / N+2 f^{N+1} / N}{(1-f)^{2}} \\
& \text { where } f=e^{-k^{2} \sigma^{2} / 6} .
\end{aligned}
$$

The resulting $\hat{S}^{G}(k)$, found numerically, indicates tighter and more ordered local packing and smaller compressibilities than resulting from $\hat{\omega}(k)$ of (24). The softening and smearing of the polymeric liquid structure in our model, compared to $\hat{S}^{G}(k)$ and to simple liquids, results from the enhanced intramolecular structure in $\hat{\omega}(k)$ already on local length scales, $1 / R_{g} \ll k<1 / \sigma$; see the inset in Fig. 1. Our model thus may claim a qualitative descriptive power for Gaussian chain polymer melts only. The extensive PRISM work reviewed in $[10,11]$ shows the power of the PRISM 


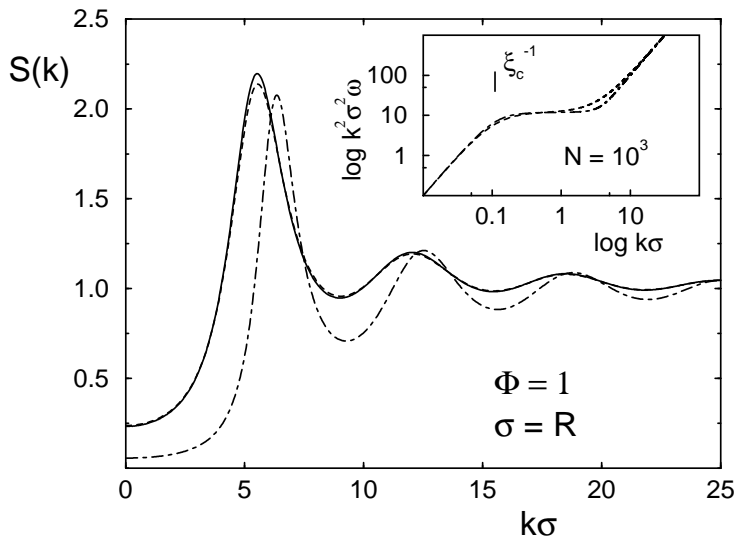

Fig. 1. Collective liquid structure factors, $\hat{S}(k)$, for melt parameters, $\Phi=1.0$ and $\Gamma=1$. The infinite molecular weight result, $1 / N=0$, is given by the solid line, the $N=10^{3}$ solution by the dashed curve. The intramolecular structure factor, $\hat{\omega}(k)$ Eq. (24), used in the evaluation is shown in a Kratky representation in the inset (dashed line) and compared to the Gaussian $\hat{\omega}^{G}(k)$ (dashed-dotted curve) for the same $N$. Its resulting $\hat{S}^{G}(k)$ is shown by a dashed-dotted curve. One finds for the reduced compressibilities $\hat{S}(0)=0.233,0.238$ and 0.057 , and for the contact values of the pair correlation function $g(R)=0.657,0.615$ and 1.284 for the infinite $N$, the $N=10^{3}$ rational $\hat{\omega}(k)$, Eq. (24), and the $N=10^{3}$ Gaussian $\hat{\omega}^{G}$, Eq. (30), results, respectively

integral equations to incorporate local chemical details and to study their consequences for the liquid structure and the observed phases. The multitude of chemistry dependent local length scales leads to a smearing of the intermolecular structure thus rendering detailed PRISM and experimental results for $\hat{S}(k)$ for ca. $k \sigma<10$ more similar to our results than to the true Gaussian $\hat{S}^{G}(k)$ following from (30) $[10,11,29]$.

\subsection{Dilute to semidilute crossover in $\Theta$-solvents}

The case of a dilute system of polymers with Gaussian intramolecular structure can be achieved by considering polymers in a $\Theta$-solvent, which is defined as a solvent where the long ranged intramolecular effects of the excluded volume interactions vanish $[1,5,28]$. This case can be achieved even for monomers which interact sterically only [25]. From Renormalization Group considerations of the $\Theta-$ point, which is identified as a tricritical point $[12,30]$, it is known that mean field like scaling laws for the collective density fluctuations hold with corrections logarithmic in the degree of polymerization [1]. In this section, a systematic expansion of the PRISM integral equations close to the $\Theta$-point is performed which leads to a scaling law of the Ornstein-Zernicke form for the collective structure factor and to another scaling law for the collective correlation length, $\xi$. Corrections of the order $\mathscr{O}(1 / N)$ are included. The range of validity of the scaling law results and material dependent corrections in lowest order in the packing fraction are identified.

In order to find the small parameters in the PRISM equations for dilute and semidilute $\Theta$-solutions, it proves convenient to introduce dimensionless variables by scaling lengths with the single polymer correlation length, $\xi_{c}=R_{g} / \sqrt{ } 2 \sim$ $\sqrt{ } N$ :

$Q(r)=1 / \xi_{c} Q^{\prime}\left(r^{\prime}\right) \quad$ where $\quad r^{\prime}=r / \xi_{c}$.

Obviously, the parameter $\tilde{g}$, Eq. (16), which has the dimension of an area is small on order $\xi_{c}^{2}$ and thus is nondimensionalized by $\tilde{g}=R^{2} \tilde{g}^{\prime}$. Importantly, one realizes that a characteristic density, $\varrho_{*}$, or packing fraction, $\Phi_{*}=$ $\pi \varrho_{*} \sigma^{3} / 6$, appears which is given by:

$\Phi_{*}=\frac{\sigma}{\xi_{c}}\left(1+\frac{1}{N}\right) \propto \frac{N}{\left(R_{g} / \sigma\right)^{3}} \propto 1 / \sqrt{ } N$,

where the proportionalities hold neglecting the $1 / N$ corrections. $\Phi_{*}$ non-dimensionalizes the packing fraction and results for fixed $\delta=\Phi / \Phi_{*}$ describe the dilute and semidilute density range. The ratio of the two microscopic length scales, the aspect ratio, $\Gamma=\sigma / R$, is the only microscopic parameter left in the rescaled equations and is also kept fixed.

The dilute and semidilute $\Theta$-solution case is described in PRISM theory in the limit, $\xi_{c} \propto \sqrt{ } N \rightarrow \infty$ and $\Phi \rightarrow 0$, such that the reduced packing fraction, $\delta=\Phi / \Phi_{*}$, is fixed. The rescaled Eqs. (A.2) to (A.7), the solvability condition, Eq. (A.1), and the formula for the liquid structure factor, Eq. (23), can be expanded in series in $R^{\prime}=R / \xi_{c}$, and the solutions, $\hat{S}\left(k^{\prime}\right)$ or $g\left(r^{\prime}\right)$, can be obtained as series in $R^{\prime}$. The calculational details are given in the appendix. A scaling law solution of the PRISM equations starting from the intramolecular structure factor, $\hat{\omega}(k)(24)$, is found:

$\hat{S}(k) \rightarrow \frac{z^{2} \xi^{2}}{1+k^{2} \xi^{2}} \quad$ for $\quad N \rightarrow \infty, \quad \Phi \rightarrow 0$,

$$
\delta=\Phi / \Phi_{*}=\text { fixed }, \quad \Gamma=\text { fixed. }
$$

The Lorentzian scaling law is universal as the microscopic parameter, the inverse length $z$ from (25), only sets an overall scale.

The correlation length, $\xi$, as a function of packing fraction obeys:

$\xi=\frac{\xi_{c}}{1+2 \Phi / \Phi_{*}}$,

and is comparable to the single polymer size only in the dilute limit. In the semidilute limit, it is much smaller and approaches the $N$-independent density screening length, $\xi_{\rho}=\sigma / 2 \Phi[15]:$

$\xi \rightarrow\left\{\begin{array}{lll}\xi_{c}=R_{g} / \sqrt{ } 2 & \text { for } & \Phi \ll \Phi_{*}, \\ \xi_{\rho}\left(1+{ }_{N}\right)={ }_{2 \Phi}\left(1+{ }_{N}^{1}\right) & \text { for } & \Phi \gg \Phi_{*} .\end{array}\right.$

Again, microscopic parameters, like the aspect ratio, $\Gamma$, have dropped out of the universal results except for the Gaussian segment size, $\sigma$, which appears in the packing fraction and the radius of gyration. The independence on the the hard core diameter, $R=\sigma / \Gamma$, has to be contrasted to the noninteracting limit, studied in Sect. 4.1. The limits leading to (29) and to (33) do not commute, as the $\Theta$-scaling laws are unaffected if $R \rightarrow 0$ is taken at the end.

The corrections of order $\mathscr{O}(1 / N)$, which are included in the asymptotic results, e.g. for the compressibility and the correlation length in the semidilute range, arise from the position of the pole of the inverse intramolecular structure factor in (12), see (25). Asymptotically they are smaller than the 
logarithmic corrections predicted by Renormalization Group arguments [1].

The intermolecular pair correlation function, $g(r)$, can be obtained easily by inverting (16) and (23):

$g(r)=1+\frac{\Phi_{*} \xi_{c}}{2 \Phi r}\left(e^{-r / \xi}-e^{-r / \xi_{c}}\right)$.

The intermolecular contributions separate into two contributions $[14,15]$. The universal correlation hole in the intermolecular density fluctuations, $h^{\mathrm{ch}}(r)=-{ }^{\sigma(1+1 / N)} e^{-r / \xi_{c}}$, exactly cancels the intramolecular long ranged correlations $[1,3,7]$. The remaining part of $g(r)$, characterized by the collective density screening length, $\xi$, describes how the compressibility and the local packing vary as a consequence of steric interactions. The Lorentzian form of $\hat{S}(k)$ and the existence of the density screening length, with $\xi_{\rho} \sim \varrho^{-1 / 2}$, had first been predicted by Edwards using a pseudopotential approach [2]. Neutron scattering experiments [13], scaling considerations using the tricritical $-\Theta$ point analogy [12], and results using the random phase approximation with three-body interactions [31], agree with the PRISM prediction $\xi_{\rho} \sim \varrho^{-1}$.

The results for $\hat{S}(k)$ and $g(r)$, which were derived here for the dilute to semidilute crossover in $\Theta$-solvents, had previously been used in more concentrated regimes $[14,15]$. In that context, it has been shown that the functional forms, Eqs. (33) and (36), and the results for $\xi_{\rho}$ are useful approximations in a wider range of densities than the presented derivation indicates $[14,15,32]$. The related "thread" and "string" approximations of Schweizer and Curro have proved valuable in a variety of more complex polymeric systems like blends and block copolymers [10, 11].

It is easily possible to find the leading corrections to the asymptotic results in order to study the range of validity of (33) and (36). Let us note the compressibility including the leading correction of order $\mathscr{O}\left(R / \xi_{c}\right)$ :

$$
\begin{aligned}
& \hat{S}(0)=z^{2} \xi^{2}\left[1-\Phi \begin{array}{cc}
2\left(1+\Phi / \Phi_{*}\right) & \Delta^{a}(\Gamma) \\
\left(1+2 \Phi / \Phi_{*}\right) & \sqrt{ } 12(1+1 / N)^{3 / 2}
\end{array}\right. \\
& \left.+\mathscr{O}\left(\left(R / \xi_{c}\right)^{2}\right)\right] \text {, }
\end{aligned}
$$

where the chemistry dependent factor $\Delta^{a}$ is given in (A.18) and equals $\Delta^{a}(1)=5.73$ for $\Gamma=1$ and infinite $N$. For fixed $\Phi / \Phi_{*}$, the corrections are of order $\mathcal{O}\left(N^{-1 / 2}\right)$.

Figure 2 shows collective structure factors for a not too large degree of polymerization, $N=10^{3}$, and at the crossover density, $\Phi=\Phi_{*} \approx 0.110$ from (32). Qualitatively, the Lorentzian form, Eq. (33), describes $\hat{S}(k)$ obtained from the full PRISM equations using (24). At the intermediate packing fraction, the non-asymptotic corrections however are large. In (37) they amount to $24 \%$ and lead to a rather close agreement of (37) with the full result for $\hat{S}(0)$ in Fig. 2 .

The appearance of universal density fluctuations in the PRISM equations is further supported by the much smaller differences of $\hat{S}(k)$ evaluated with $\hat{\omega}(k)$ of (24) and of (30) in the semidilute than in the melt case. In Fig. 2 both static structure factors are close for ca. $k \xi<1.5$. The insets in Fig. 1 and 2 compare the two intramolecular structure factors $\hat{\omega}(k)$ entering into the PRISM calculations. Comparing $\hat{S}(k)$ and $\hat{\omega}(k)$ in Fig. 2 and the inset, one notices the strong reduction in magnitude of the density fluctuations and of the

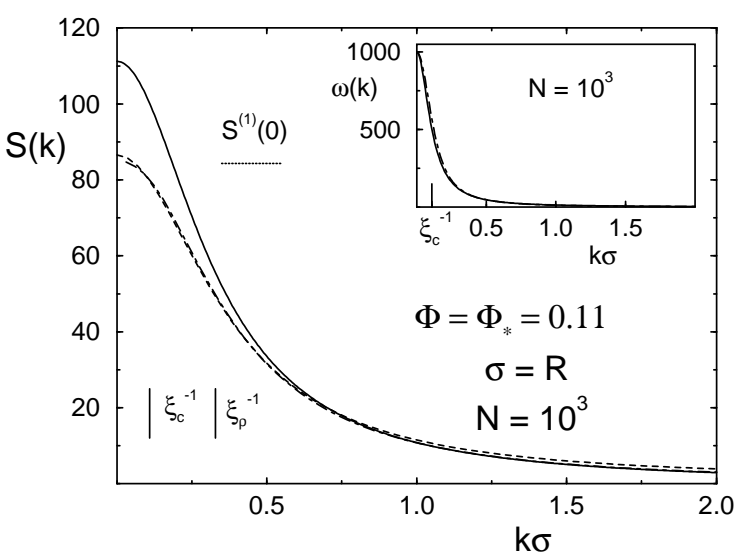

Fig. 2. Collective liquid structure factors, $\hat{S}(k)$, for a semidilute $\Theta$ solution, $\Phi=\Phi_{*}=0.110, N=10^{3}$, and $\Gamma=1$. The scaling law, (33), is given by the solid line, and the compressibility including the leading correction, Eq. (37), is indicated by a horizontal bar. Solutions for $\hat{S}(k)$ (dashed) using $\hat{\omega}(k)$, Eq. (24), and $\hat{S}^{G}$ (dot-dashed) using $\hat{\omega}^{G}$, Eq. (30), are compared for these parameters. The inset shows the corresponding intramolecular structure factors, $\hat{\omega}(k)$ (solid) and $\hat{\omega}^{G}$ (dot-dashed). One finds for the reduced compressibilities $\hat{S}(0)=111.2,84.31,86.65$ and 84.7 , and for the contact values of the pair correlation function $g(R)=0 ., 0.0375,0.0424$ and 0.0394 for the scaling law, Eqs. (33) and (36), including the first order correction, Eqs. (37) and (38), the rational $\hat{\omega}(k)$, Eq. (24), and the Gaussian $\hat{\omega}^{G}$, Eq. (30), results, respectively

corresponding correlation length of the collective relative to the single polymer fluctuations.

The contact value of the pair correlation function, which vanishes in the asymptotic limit, can also be found in lowest order in $R / \xi_{c}$ :

$g(R)=\frac{R}{\xi_{c}}\left(1+\Phi / \Phi_{*}\right) \Delta^{g}(\Gamma)+\mathscr{O}\left(\left(R / \xi_{c}\right)^{2}\right)$,

where the chemistry dependent factor, $\Delta^{g}$, is given in (A.23) and equals $\Delta^{g}(1)=0.171$ for $\Gamma=1$ and infinite $N$. For the parameters used in Fig. 2, the lowest order value, $g(R)=$ 0.0375 from (38), differs by $13 \%$ from the full value, $g(R)=$ 0.0424 . In the semidilute density range, $\Phi \gg \Phi_{*}$, the contact value increases linearly [32] with packing fraction, $g(R)=$ $c \Phi$, where the constant $c$ increases from ca. 0.08 to 0.3 with aspect ratio $\Gamma$ increasing from 0.5 to 1.5 .

\section{Discussion}

A reformulation of the PRISM integral equations using the Wiener-Hopf factorization as pioneered by Baxter has been presented. It is expected that it proves useful in situations where a wide variation of relevant length scales slows down direct numerical solutions of the integral equations given by (1) to (4). It is especially of interest if simple expressions for the intramolecular structure factor can be chosen, and then allows analytical solutions of the PRISM equations. Various long wave length correlations in polymeric liquids should be amenable in this framework. Studies of concentrated colloidal suspensions with added non-adsorbed polymers are under way [33], where previously PRISM studies [34] have been encumbered by the large difference in colloidal and monomeric diameters. 
A simple example for the use of the reformulation of the PRISM equations is given by the study of Gaussian homopolymer systems. Note that the solved PRISM model in an unified way describes microscopic packing effects, as dominant in the dense melt case, see Fig. 1, and more universal mesoscopic density fluctuations, as appear in the semidilute $\Theta$-solution case, see Fig. 2. The correlations in the studied polymeric liquid only arise from entropic intraand intermolecular terms. The justification of such a model has been discussed in Sect. 4.

A major restriction of the presented most simple version of PRISM theory for the study of polymer solutions is the assumption of a given intramolecular structure. Extensions of liquid state integral equation theories to determine intra- and intermolecular correlations together have been formulated, e.g. in $[35,36]$. Within the PRISM approach quantitative results for e.g. the variation of the radius of gyration with concentration have been obtained and compared to computer simulation results [37].

In the studied PRISM model of Gaussian homopolymer systems, it has been shown that for flexible polymers, $2 / 3<$ $\Gamma<3 / 2$, no spinodal points occur. At melt like densities, rather small compressibilities result with the local packing familiar from simple liquids [10, 24]. Material independent scaling laws, of Ornstein-Zernicke form for the structure factor, are predicted for the dilute to semidilute crossover in $\Theta$-solvents. This result agrees with Renormalization group calculations [12] and experimental observations [13]. The characteristic density, $\varrho_{*} \sim N / R_{g}^{3} \sim 1 / \sqrt{ } N$, appears and the scaling laws hold in the limit $\Phi_{*} \sim \sigma^{3} \varrho_{*} \ll 1$. Corrections to the scaling law description have been given, e.g. for the compressibility and the local contact value of the intermolecular pair correlation function, and are generally of linear order in the packing fraction in the semidilute concentration range.

The origin of the material independent scaling law for the collective density fluctuations can be recognized by considering $\hat{S}^{-1}(k)=\hat{\omega}^{-1}(k)-\varrho \hat{c}(k)$, Eq. (2), in the specified limit. $\hat{\omega}^{-1}(k)$ is a small number for $k R_{g} \ll 1$ and increases quadratically for intermediate wave vectors, $\hat{\omega}^{-1}(k) \sim k^{2}$ for $1 / R_{g} \ll k \ll 1 / \sigma$. The direct correlation function, $\hat{c}(k)$, takes some (negative) constant value for $k R \ll 1$. Outside the specified wave vector ranges, both functions vary non-universally, and $\hat{\omega}^{-1}$ crosses over to 1 while $\hat{c}(k)$ drops to zero. The specified scaling law limit now consists of, infinitely extending the wave vector interval, $1 / R_{g} \ll k \ll 1 / \sigma$, so that the solutions, $\hat{S}(k)$ and $\hat{c}(k)$ are determined in this range of universal behavior.

The success of PRISM theory to describe the dilute and semidilute $\Theta$-solution cases may come as a small surprise as it is well documented that the small molecule RISM equations become inaccurate for small densities [24]. In the present case, however, the PRISM results, e.g. for the osmotic pressure, $\Pi$, as calculated from the compressibility, $\beta \Pi=\int_{0}^{\rho} d \bar{\rho} \hat{S}^{-1}(k=0, \bar{\rho})$, compare well to the known results $[1,10,11,12], \beta \Pi \rightarrow \varrho / N$ for $\Phi \ll \Phi_{*}$ and $\beta \Pi \rightarrow(12 / \pi) / \xi_{\rho}^{3}$ for $\Phi \gg \Phi_{*}$, where $\xi_{\rho}=\sigma / 2 \Phi$.

In this context it is of interest that recently Chandler has derived the PRISM scaling laws, Eqs. (33) and (36), in a Gaussian field theory, where the Gaussian field is excluded from a rigid manifold [38]. The studied PRISM model thus appears as an extension of a Gaussian field theoretical model which incorporates finite microscopic length scales. It describes the crossover from the universal density fluctuations close to the $\Theta$-point, which are amenable also by field theoretic means, to the locally dominated liquid structure, present at high densities. As PRISM thus achieves a description of low density polymeric systems, it appears promising to extend the presented PRISM approach to colloid polymer mixtures [33], where recent experiments show varying colloidal phase diagrams in dependence on the ratio of colloidal diameter to polymer radius of gyration [39]. Those features of the colloid-polymer phase diagram which differ from the phase diagram of binary colloid mixtures with identical ratios of their diameters, are of interest, as they have to originate from the internal polymer structure.

I am greatly indebted to Kenneth Schweizer for many enlightening discussions about liquid state theories. I cordially thank Edwin David for providing the programs to solve the full PRISM equations numerically, Thomas Franosch for getting me started with symbolic computations, and Kenneth Schweizer for a critical reading of the manuscript. Partial financial support by the Deutsche Forschungsgemeinschaft under grant Fu 309/1-1, and the United States National Science Foundation MRSEC program via grant number NSF-DMR-89-20538, are acknowledged.

\section{Appendix}

Details of the solution of the PRISM equations for homopolymer systems with the intramolecular structure of (24) are given.

The solvability condition of the model follows from (15) and (26):

$(1-\tilde{Q}) d=\frac{z^{2} \xi_{c}^{2}-1}{2 z \xi_{c}^{2}}$.

Inserting the form of $Q(r)$ from (10), (26) and (28) into (17) leads to five linear equations for the six unknown coefficients $a, \ldots, f$ :

$$
\begin{aligned}
& a=\frac{2 \pi \varrho}{\left(z \xi_{c}\right)^{2}}\left(1-\int_{0}^{\infty} d s Q(s)\right), \\
& b=\frac{2 \pi \varrho}{\left(z \xi_{c}\right)^{2}} \int_{0}^{\infty} d s s Q(s), \\
& c=-\frac{z^{2} \xi_{c}^{2}-1}{\left(z \xi_{c}\right)^{2}} \pi \varrho \tilde{g} d,
\end{aligned}
$$

$$
\begin{aligned}
& -\left(\xi_{c}+R\right) a-b+\frac{z e^{-z R}}{z \xi_{c}+1} e+\frac{z e^{z R}}{z \xi_{c}-1} f \\
& +e^{-z R}\left(\frac{z R}{z \xi_{c}+1}-\frac{1}{\left(z \xi_{c}+1\right)^{2}}\right) c=0
\end{aligned}
$$

$$
\begin{aligned}
\left(\xi_{c}+R / 2\right) & a+\left(2 \xi_{c}+R\right) b+\left(\frac{e^{-z R}}{z \xi_{c}+1}+\frac{1}{z \xi_{c}-1}\right) e \\
& -\left(\frac{e^{z R}}{z \xi_{c}-1}+\frac{1}{z \xi_{c}+1}\right) f+\left(e ^ { - z R } \left(\frac{R}{z \xi_{c}+1}\right.\right. \\
& \left.\left.+\frac{\xi_{c}}{\left(z \xi_{c}+1\right)^{2}}\right)+\frac{\xi_{c}}{\left(z \xi_{c}-1\right)^{2}}\right) c+\frac{1}{z \xi_{c}-1} d=1 / \xi_{c} .
\end{aligned}
$$


Inserting $Q(r)$ into (19) gives the sixth linear equation:

$(1-\tilde{Q}) \pi \varrho \tilde{g}=\frac{z^{2} \xi_{c}^{2}}{z^{2} \xi_{c}^{2}-1} f$.

Equations (A.2) to (A.5) can be solved for $a, b, e, f$ depending on the parameters $c, d$. Using Eq. (A.6), Eq. (A.7) then turns into a linear equation for $d$ of the form: $\left(x+x^{\prime} \tilde{g}+\right.$ $\left.x^{\prime \prime} d\right) \tilde{g}=y+y^{\prime} \tilde{g}+y^{\prime \prime} d$, while $\tilde{Q}$ is of the form $z+z^{\prime} \tilde{g}+z^{\prime \prime} d$, where the $x, y, z$ denote some constants depending on $N$, $R, \sigma$ and $\varrho$. Solving Eq. (A.7) for $d$ and using the form of $\tilde{Q}$ in (A.1) then leads to a quartic solvability condition for $\tilde{g}$ as claimed.

In the special limits discussed in Sect. 4.1 to 4.3 simplifications are possible.

\section{A.1. Non-interacting case}

For $R=0$, but finite $\sigma, \varrho$ and $N$, the parameter $\tilde{g}$ and the coefficients of the factor function can be found easily:

$$
\begin{aligned}
& \tilde{g}=\frac{1}{z^{2}} \\
& a=\frac{2 \pi \varrho}{\left(z \xi_{c}\right)^{3}}, \\
& b=\frac{2 \pi \varrho \xi_{c}}{\left(z \xi_{c}\right)^{3}}\left(1-\frac{1}{z \xi_{c}}\right), \\
& c=-\frac{\pi \varrho}{z}\left(1-\left(\frac{1}{z \xi_{c}}\right)^{2}\right)\left(1-\frac{1}{z \xi_{c}}\right), \\
& d=z\left(1-\frac{1}{z \xi_{c}}\right), \\
& e=-\frac{\pi \varrho}{2 z^{2}}\left(1-\frac{2}{z \xi_{c}}-\frac{7}{\left(z \xi_{c}\right)^{2}}\right)\left(1-\frac{1}{z \xi_{c}}\right), \\
& f=\frac{\pi \varrho}{2 z^{2}}\left(1-\left(\frac{1}{z \xi_{c}}\right)^{2}\right)\left(1+\frac{1}{z \xi_{c}}\right),
\end{aligned}
$$

\section{A.2. Infinite degree of polymerization}

In the limit of $1 / N=0, q(r)$ is given by (28), where the hard sphere coefficients vanish, $a=b=0$. The remaining coefficients are found from (A.4) to (A.7) when (A.2) and (A.3) are inserted. The linear equations can be solved symbolically leading to a quartic equation for $\tilde{g}$, as in the general case, which is simplified somewhat if it is turned into an equation for $\tilde{Q}$. The known solution in the non-interacting case, $\tilde{Q}=1 / 2$, allows to identify the physical solution for finite $\Phi$. As the equation or its solution are too lengthy to report, Fig. 3 presents the roots $1-\tilde{Q}$ as functions of the packing fraction for some aspect ratios typical of flexible polymers. The curves neither collapse if plotted versus $\varrho R^{3}$. A divergent susceptibility corresponds to $1-\tilde{Q}(0)=0$, which gives a quadratic equation for $Q$. Its physical root, again approaching $1 / 2$ for $\Phi \rightarrow 0$, is included in Fig. 3. The curves of the roots $\tilde{Q}$ from the solvability condition and from the divergence of the compressibility do not cross for finite packing fractions. Therefore, PRISM predicts finite compressibilities

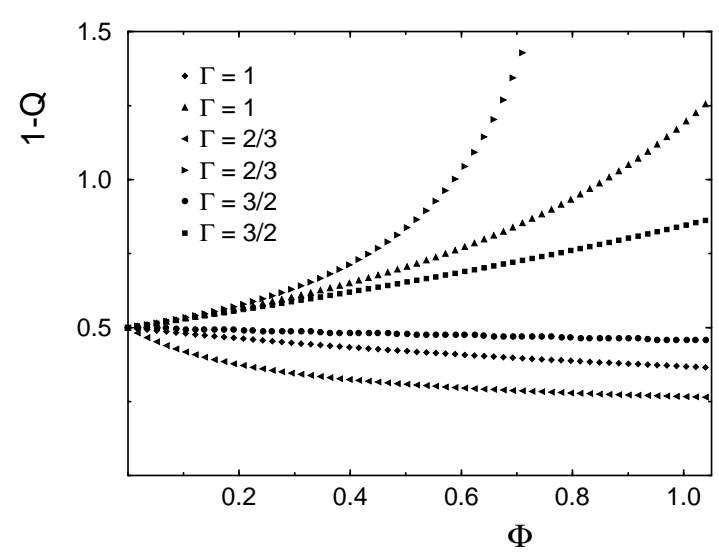

Fig. 3. Roots $1-\tilde{Q}$ of the solvability condition (lower 3 curves) and of the equation $\tilde{Q}(0)=1$, where the compressibility diverges (upper 3 curves), for the indicated 3 values of the aspect ratio

in homopolymer systems of flexible polymers at finite densities. The divergence of the intramolecular structure factor, $\hat{\omega}(k) \rightarrow \infty$ for $k \rightarrow 0$ is cancelled exactly except for at $\Phi=0$.

\section{A.3. Dilute to semidilute crossover at $\Theta$-temperature}

The dimensionless variables introduced in Sect. 4.3 and an

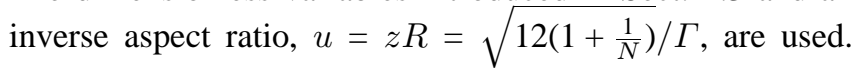
Equations (A.2) to (A.7) can be written as matrix equation, $A \mathbf{x}=\mathbf{b}$, where $\mathbf{x}^{T}=\left(a^{\prime}, \ldots, f^{\prime}\right)$, and the $6 \times 6$ matrix $A$ can be expanded in a series in $R^{\prime}, A=A_{0}+A_{1} R^{\prime}+\cdots$; likewise for $\mathbf{b}$. As $A_{0}$ is singular, the solution vector $x$ has to be expanded like $\mathbf{x}=\frac{1}{R^{\prime}} \mathbf{x}_{-1}+\mathbf{x}_{0}+\cdots$. The hierarchy of matrix equations,

$$
\sum_{n=0} R^{\prime n-1}\left[\sum_{m=0}^{n} A_{n-m} \mathbf{x}_{m}-\mathbf{b}_{n-1}\right]=0,
$$

can be solved as $A_{0}$ has one left (right) eigenvector $e(\hat{e})$, where $e^{T}=(0,0,0,1,0,0)$ and $\hat{e}=(0,-2,0,0,1,0)$, respectively, belonging to the zero eigenvalue and the condition for (A.15) to have a solution at step $n$, therefore, runs:

$\hat{e} \cdot \sum_{m=0}^{n-1}\left[A_{n-m} \mathbf{x}_{m}-\mathbf{b}_{n-1}\right]=0$.

In order to find $\tilde{g}^{\prime}$ to lowest order in $R^{\prime}$ it is necessary to find $\mathbf{x}\left(R^{\prime}\right)$ up to linear order in $R^{\prime}$, as the solvability condition, Eq. (A.1), has to be formulated correctly including order $\left(R^{\prime}\right)^{2}$. Then it is quadratic in $\tilde{g}$ and the physical solution is: $\tilde{g}^{\prime}=R^{\prime}(1+\delta) \frac{8 e^{u}\left(1+e^{2 u}\right)}{u^{4}\left(e^{4 u}-1-4 u e^{2 u}\right)}+\mathcal{O}\left(\left(R^{\prime}\right)^{2}\right)$.

For the scaling law solutions, Eqs. (33) and (34), it suffices to show $\tilde{g} \propto R^{\prime}$. The coefficients of $Q(r)$ follow where those orders in $R^{\prime}$ are given which are required in Sect. 4.3:

$$
\begin{gathered}
a^{\prime}=\frac{R^{\prime}(1+\delta)}{u}\left[1+\frac{\delta R^{\prime}(1+\delta)}{u(1+2 \delta)} \Delta^{a}+\mathcal{O}\left(\left(R^{\prime}\right)^{2}\right)\right], \\
\text { where } \Delta^{a}=\frac{4\left(2+u+4\left(1+u^{2}\right) e^{2 u}+(2-u) e^{4 u}\right)}{\left(1-e^{4 u}+4 u e^{2 u}\right)},
\end{gathered}
$$


$b^{\prime}=\frac{R^{\prime} \delta}{u}+\mathscr{O}\left(\left(R^{\prime}\right)^{2}\right)$,

$c^{\prime}=-\delta(1+\delta) \frac{4 e^{u}\left(1+e^{2 u}\right)}{\left(e^{4 u}-1-4 u e^{2 u}\right)}+\mathscr{O}\left(R^{\prime}\right)$,

$d^{\prime}=\frac{u}{R^{\prime}}-(1+2 \delta)+\odot\left(R^{\prime}\right)$,

$e^{\prime}=-R^{\prime} \delta(1+\delta) \frac{2 e^{u}\left(1+e^{2 u}\right)(1-2 u)}{u\left(e^{4 u}-1-4 u e^{2 u}\right)}+\mathscr{Q}\left(\left(R^{\prime}\right)^{2}\right)$

$f^{\prime}=R^{\prime} \delta(1+\delta) \frac{2 e^{u}\left(1+e^{2 u}\right)}{u\left(e^{4 u}-1-4 u e^{2 u}\right)}+\mathscr{\sigma}\left(\left(R^{\prime}\right)^{2}\right)$.

With these results the contact value follows from (18):

$$
\begin{aligned}
g(R)= & R^{\prime}(1+\delta) \Delta^{g}+\mathscr{O}\left(\left(R^{\prime}\right)^{2}\right), \\
& \text { where } \Delta^{g}=\frac{2\left(e^{4 u}-1+4 u e^{2 u}\right)}{u^{2}\left(e^{4 u}-1-4 u e^{2 u}\right)} .
\end{aligned}
$$

\section{References}

1. de Gennes, P.G.: Scaling Concepts in Polymer Physics. Ithaca: Cornell University Press 1979

2. Edwards, S.F.: Proc. Phys. Soc. (London) 88, 265 (1966)

3. de Gennes, P.G.: J. Phys. (Paris) 31, 325 (1970)

4. Oono, Y.: Adv. Chem. Phys. 61, 301 (1985)

5. Flory, P.J.: Principles of polymer chemistry. Cornell University Press: Ithaca 1953

6. Dudowicz, J., Freed, K.: Macromolecules 24, 5112 (1991); J. Chem. Phys. 100, 4653 (1994)

7. Schweizer, K.S., Curro, J.G.: Phys. Rev. Lett. 58, 246 (1987)

8. Chandler, D., Andersen, H.C.: J. Chem. Phys. 57, 1930 (1972)

9. Curro, J.G., Schweizer, K.S., Grest, G.S., Kremer, K.: J. Chem. Phys. 91, 1357 (1989); Kremer, K., Grest, G.S.: J. Chem. Phys. 92, 5057 (1990)

10. Schweizer, K.S., Curro, J.G.: Adv. Polym. Sci 116, 319 (1994)

11. Schweizer, K.S., Curro, J.G.: Adv. Chem. Phys. 98, in press (1996)

12. Daoud, M., Jannink, G.: J. Phys. (Paris) 37, 973 (1976)

13. Cotton, J.P., Nierlich, M., Boue, F., Daoud, M., Farnoux, B., Jannink, G., Duplessix, R., Picot, C.: J. Chem. Phys. 65, 1101 (1976)

14. Schweizer, K.S., Curro, J.G.: Macromolecules 21, 3070, 3082 (1988)
15. Schweizer, K.S., Curro, J.G.: Chem. Phys. 149, 105 (1990)

16. Baxter, R.J.: Austral. J. Phys. 21, 563 (1968); J. Chem. Phys. 52, 4559 (1970)

17. Blum, L.: Mol. Phys. 30, 1529 (1975); Blum, L., Høye, J.S.: J. Stat. Phys. 19, 317 (1978)

18. Høye, J.S., Blum, L.: J. Stat. Phys. 16, 399 (1977)

19. Morris, G.P., Perram, J.W., Smith, E.R.: Mol. Phys. 38, 465 (1979); Morris, G.P., Smith, E.R.: J. Stat. Phys. 24, 607 (1981)

20. Fishman, S., Fisher, M.E.: Physica 108A, 1 (1981)

21. Cummings, P.T., Stell, G.: J. Chem. Phys. 78, 1917 (1983)

22. Hafner, J., Jank, W.: Z. Phys. B 70, 81 (1988)

23. Konior, J., Jedrzejek, C.: Z. Phys. B 80, 125 (1990)

24. Hansen, J.P., McDonald, I.R.: Theory of Simple Liquids. London: Academic Press 1986

25. Frenkel, D., Louis, A.A.: Phys. Rev. Lett. 68, 3363 (1992); Dijkstra, M., Frenkel, D., Hansen, J.P.: J. Chem. Phys. 101, 3179 (1994)

26. Yethiraj, A., Schweizer, K.S.: J. Chem. Phys. 97, 5927 (1992); Schweizer, K.S., Yethiraj, A.: J. Chem. Phys. 98, 9053 (1993)

27. Schweizer, K.S., David, E.F.. Singh, C., Curro, J.G., Rajasekaran, J.J.: Macromolecules 28, 1528 (1995)

28. Doi, M., Edwards, S.F.: The Theory of Polymer Dynamics. Oxford: Clarendon 1986

29. Honnell, K.G., McCoy, J.D., Curro, J.G., Schweizer, K.S., Narten, A.H., Habenschuss, A.: J. Chem. Phys. 94, 4659 (1991); Narten, A.H., Habenschuss, A., Honnell, K.G., McCoy, J.D., Curro, J.G., Schweizer, K.S.: J. Chem. Soc. Faraday Trans. 88, 1791 (1992)

30. de Gennes, P.G.: J. Phys. (Paris) Lett. 36, L55 (1975)

31. Muthukumar, M.: J. Chem. Phys. 85, 4722 (1986)

32. Schweizer, K.S., Szamel, G.: J. Chem. Phys. 103, 1934 (1995)

33. Fuchs, M., Schweizer, K.S.: in preparation (1997)

34. Yethiraj, A., Hall, C.K., Dickmann, R.: J. Colloid Interface Sci. 151, 102 (1992)

35. Genz, U., Klein, R.: J. Phys. (Paris) 50, 439 (1989); Genz, U., Klein, R., Benmouna, M.: J. Phys. (Paris) 50, 449 (1989)

36. Grayce, C.J., Schweizer, K.S.: J. Chem. Phys. 100, 6846 (1994); Melenkevitz, J., Curro, J.G., Schweizer, K.S.: J. Chem. Phys. 99, 5571 (1993)

37. Grayce, C.J., Yethiraj, A. Schweizer, K.S.: J. Chem. Phys. 100, 6857 (1994)

38. Chandler, D.: Phys. Rev. E 48, 2898 (1993)

39. Poon, W.C.K., Selfe, J.S., Robertson, M.B., Ilett, S.M., Pirie, A.D., Pusey, P.N.: J. Physique II 3, 1075 (1993); Pusey, P.N., Poon, W.C.K., Ilett, S.M., Bartlett, P.: J. Phys. : Condens. Matter 6, A29 (1994); Ilett, S.M., Orrock, A., Poon, W.C.K., Pusey, P.N.: Phys. Rev. E 51, 1344 (1995) 